\title{
Détermination Des Teneurs En Mercure (HG) Total Chez La Moule Perna Perna Et L'oursin Echinometra Lucunter De La Baie De Soumbédioune (Sénégal) Et Évaluation Des Risques Sanitaires
}

\author{
Ousmane Diankha, \\ Laboratoire de Physique de l'Atmosphère et de l'Océan (LPAO-SF), Ecole \\ supérieure polytechnique, Université Cheikh Anta Diop (UCAD),Fann, \\ Dakar, Sénégal \\ Direction des Aires Marines Communautaires Protégées, Ministère de \\ l'Environnement et du Développement Durable, Fann, Dakar, Sénégal

\section{Thiapato Ndiaye, \\ Constance Agbogba,} \\ Département de Biologie Animale, Faculté des Sciences et Techniques, \\ Université Cheikh Anta Diop (UCAD), Fann, Dakar, Sénégal

\section{Alassane Sarr,} \\ Institut Universitaire de Pêche et d'Aquaculture (IUPA), Université Cheikh \\ Anta Diop UCAD II bâtiment pédagogique/Rez-de-chaussée, Dakar
}

\begin{abstract}
Soumbedioune Bay, marked by an intense activity of exploitation and marketing of seafood, is the receptacle of huge quantities of wastewater from channel 4 and artisanal activities which are carried out there. That's why a study aiming to determine the level of mercury contamination of mussels (Perna perna) and sea urchins (Echinometra lucunter) and to assess the health risks associated with their consumption was performed. It consisted to collect samples (non-transformed and transformed products) of these two species during the periods of abundance and non-abundance in 2018. The direct mercury analyzer DMA-80 was used to determine the mercury contents contained in these species. The results obtained after analyzes show the presence of this very toxic metal in the edible parts of these species but at concentrations below the standard established by the American agency for the protection of the environment. These concentrations vary between 0.0172 and 0.0475 mg.kg-1 for mussels against 0.0153 and $0.0274 \mathrm{mg} . \mathrm{kg}-1$ for sea urchins. The health risk indices obtained (oscillating between 0.070 and 0.844 ) less than 1 reveal that the consumption of these species remains safe for the
\end{abstract}


moment. However, they should be consumed moderately due to the ability of mercury to bioaccumulate in organisms.

Keywords: Bioaccumulation, Health Risk, Mercury, Mussel, Sea Urchin, Senegal

\title{
Determination of Total Mercury (HG) Concentrations in the Mussel Perna Perna and the Sea Urchin Echinometra Lucunter From Soumbédioune Bay (Senegal) and Health Risk Assessment
}

\begin{abstract}
Ousmane Diankha,
Laboratoire de Physique de l'Atmosphère et de l'Océan (LPAO-SF), Ecole supérieure polytechnique, Université Cheikh Anta Diop (UCAD),Fann,

Dakar, Sénégal

Direction des Aires Marines Communautaires Protégées, Ministère de l'Environnement et du Développement Durable, Fann, Dakar, Sénégal

\section{Thiapato Ndiaye, Constance Agbogba,}

Département de Biologie Animale, Faculté des Sciences et Techniques, Université Cheikh Anta Diop (UCAD), Fann, Dakar, Sénégal

\section{Alassane Sarr,}

Institut Universitaire de Pêche et d'Aquaculture (IUPA), Université Cheikh Anta Diop UCAD II bâtiment pédagogique/Rez-de-chaussée, Dakar
\end{abstract}

\section{Résumé}

La baie de Soumbédioune où s'effectue une importante activité de cueillette et de commercialisation de fruits de mer, constitue le réceptacle d'énormes quantités d'eaux usées et de déchets provenant du canal 4 et des activités artisanales qui s'y effectuent. C'est dans ce contexte qu'une étude visant à déterminer le niveau de contamination par le mercure des moules (Perna perna) et des oursins (Echinometra lucunter) et à évaluer les risques sanitaires liés à consommation de ces produits a été réalisée. Elle a consisté à collecter des échantillons (crus et transformés) de ces deux espèces durant les périodes d'abondance et de non-abondance en 2018. L'analyseur direct de mercure DMA-80 a été utilisé pour déterminer teneurs en mercure contenues dans ces espèces. Les résultats obtenus après analyses montrent la présence de 
ce métal très toxique dans les parties comestibles de ces espèces mais à des concentrations en deçà de la norme établie par l'agence américaine pour la protection de l'environnement. Ces concentrations varient entre 0,0172 et 0,0475 mg.kg-1 pour les moules contre 0,0153 et $0,0274 \mathrm{mg} . \mathrm{kg}-1$ pour les oursins. Les indices de risque sanitaire obtenus (oscillant entre 0,070 et 0,844 ) sont inférieurs à 1 révèlent que la consommation de ces espèces reste pour le moment sans danger. Néanmoins, elles devraient être consommées modérément en raison de la capacité du mercure à se bioaccumuler dans les organismes.

Mots clés: Bioaccumulation, Mercure, Moule, Oursin, Risque Sanitaire, Sénégal

\section{Introduction}

Le mercure est un métal trace toxique, reconnu de nos jours comme un polluant important et néfaste pour l'Homme et l'environnement (Marusczak, 2010). Il est présent naturellement dans les eaux mais à de très faibles concentrations (Scheuhammer et al., 2012). Cependant, à travers l'industrialisation, l'exploitation artisanale de l'or et de l'argent, les rejets de déchets d'amalgames dentaires entre autres, l'homme contribue à l'augmentation de la quantité de mercure dans les océans (Bell et al., 2014 ; Ndiaye, 2019). Des études récentes ont révélé que la quantité de mercure dans les océans a connu une hausse depuis l'ère industrielle (Marusczak, 2010 ; Bell et al., 2014).

Le danger lié à l'exposition au mercure relève du fait de sa capacité à se bioaccumuler à travers les divers échelons des chaînes trophiques marines jusqu'au consommateur final (Metongo, 1991), entraînant ainsi un phénomène de bioamplification (Marusczak, 2010 ; Noel et al., 2011 ; Diop et al., 2016). Au Japon, le scandale des eaux de la baie de Minamata avec l'intoxication de pécheurs suite à la contamination de produits halieutiques contaminés au mercure ou encore l'évènement tragique des céréales contaminés en Iraq témoigne des effets néfastes du mercure pour l'homme notamment dans sa forme organique appelé méthylmercure (MEJ, 2013). Malgré ces terribles évènements, la circulation du mercure dans l'environnement n'est surveillée que très peu dans certains pays en particuliers les pays pauvres.

Au Sénégal, la zone littorale fait l'objet d'une grande agglomération et d'une intense prolifération des activités industrielles (CCLME, 2014 ; ANSD, 2019). Les rejets urbains et industriels associés à ceux d'origine inconnue constituent à bien des égards la principale source de pollution dans la capitale sénégalaise, notamment dans la baie de Soumbédioune où les activités polluantes sont pratiquées à proximité de la baie et rejettent directement leurs effluents dans l'océan (Diop et al., 2017 ; Ndiaye, 2019). Les études 
consacrées à la contamination au mercure des produits halieutiques sont assez rares au Sénégal. A ce sujet on peut citer les travaux de Diop et al., (2017) qui ont étudié la contamination et la bioaccumulation de métaux traces dans les moules, les crevettes et les poissons le long de la côte sénégalaise, Soumbédioune y compris. Diouf (2017) a montré la présence du mercure dans les huitres et arches au niveau de l'aire marine protégée de Joal-Fadiouth.

De l'examen de l'ensemble de ces travaux, il ressort nettement que la pollution au mercure du littoral sénégalais constitue un problème écotoxicologique préoccupant pour le milieu marin et pourrait être à l'origine d'un réel problème de santé publique pour le Sénégal, un pays où la consommation des produits halieutiques constitue une source importante de protéines animales (Diop et al., 2017). Face cette situation et aux vues des connaissances actuelles à ce sujet, cette étude contribue à améliorer les connaissances sur le niveau de contamination par le mercure de la côte sénégalaise. Ceci à travers l'évaluation des teneurs en mercure de la moule (Perna perna) et de l'oursin (Echinometra lucunter) qui sont fortement commercialisés au niveau de Soumbédioune. Elle consiste à déterminer les variations temporelles (période d'abondance et période de non abondance) des teneurs en mercure dans les produits crus et grillés chez les deux espèces et estimer les risques sanitaires liés à leur consommation.

Le choix porté sur ces deux espèces se justifie par leur forte demande sur le marché national en plus du fait qu'ils constituent de bons bioindicateurs de la pollution des eaux. A cela s'ajoute la grande sensibilité qu'offrent ces deux espèces comparée aux techniques d'analyses chimiques (Augier et al., 1997). En ce qui concerne la moule, elle constitue l'un des bioindicateurs le plus utilisés en écotoxicologie (Augier et al., 1997). Cependant l'utilisation conjointe des moules et des oursins dans une même étude offre des avantages car les deux sont complémentaires. La moule concentre directement les polluants à partir de l'eau de mer. Elle est aussi capable de capter les polluants en suspension, véhiculés par les particules retenues par les branchies (Augier et al., 1997). Quant à l'oursin, sa contamination peut provenir d'un transfert alimentaire par l'intermédiaire des algues dont il se nourrit, de même que des matières organiques dissoutes absorbées par voie tégumentaire (Régis, 1978 in Augier et al., 1997).

\section{Matériel et méthodes}

Zone d'étude

L'étude est réalisée dans la baie de Soumbédioune localisée dans le département de Dakar et située à $11 \mathrm{~m}$ d'altitude derrière la route de la Corniche Ouest (Figure 1). Sur le plan économique, elle est l'un des principaux centres de pêche du Sénégal et assure l'approvisionnement en fruits de mer du quai de Soumbédioune en l'occurrence les moules et les oursins. En 
dépit de ces potentialités, la baie de Soumbédioune est soumise à une pollution du fait de divers rejets d'origine anthropique (Ndiaye, 2019). En effet, la baie de Soumbédioune est le réceptacle des rejets urbains du Canal Ouest (Canal 4) qui draine les eaux usées non épurées issues des hôpitaux et des habitations domestiques d'une bonne partie de la ville de Dakar. Elle constitue également par le biais du village artisanal le siège de certaines activités comme l'extraction artisanale de l'or et de l'argent par le mercure et l'utilisation de peintures antifongiques pour la sculpture, alors que les sites de collecte des moules et des oursins sont localisés dans les îles Madeleines, situées entre deux et trois km de la baie (Figure 1 B).

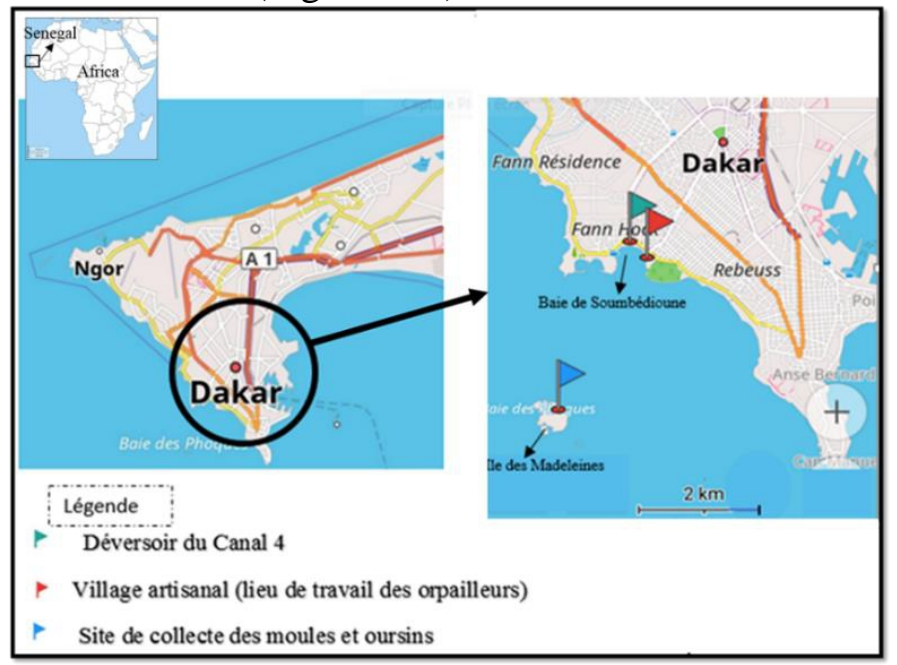

Figure 1. Site d'étude. A : localisation de la Baie de Soumbédioune, des différents points d'émissions de mercure et du site de collecte des moules et des oursins.

\section{Collecte du matériel biologique}

La collecte des échantillons a eu lieu au niveau du quai de Soumbédioune en périodes d'abondance et de non-abondance (Avril et Décembre) 2018 auprès des collecteurs et vendeurs de moules et d'oursins (Figure $2 \mathrm{AB}$ ). Deux types de produits ont été échantillonnés : les produits crus et les produits grillés. Chez les moules, les adultes dont la longueur de la coquille est comprise entre 8 et $10 \mathrm{~cm}$ sont ciblés. Chez les oursins, les adultes dont le diamètre est compris entre 8 et $10 \mathrm{~cm}$ sont ciblés. Au total 120 individus dont 60 ( 30 crus et 30 grillés) et 60 individus d'oursins ( 30 crus et 30 grillés) ont été collectés. Il a consisté à collecter pour chaque période 15 individus de moules crues et 15 moules grillées, et 15 oursins crus et 15 grillés. Après La collecte, les moules et oursins, bien emballés dans des sachets en 
plastique étiquetés pour éviter toute contamination sont acheminés directement au laboratoire pour l'analyse.

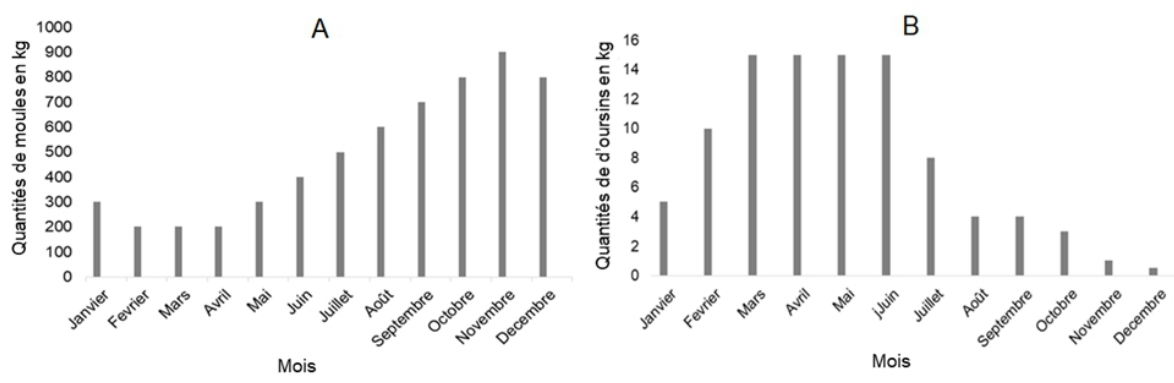

Figure 2 : Evolution mensuelle des quantités de moules et d'oursins au niveau de Soumbédioune

\section{Traitement du matériel biologique}

Le traitement du matériel biologique a été effectué au laboratoire. Ce travail est réalisé à l'œil nu dans les conditions optimales et s'est déroulé en plusieurs étapes

\section{Dissection}

Elle est réalisée avec différents instruments de laboratoire afin d'isoler les parties consommables dans les différents lots d'oursins et de moules. Les parties consommées correspondent aux gonades chez les oursins et à toute la partie molle (masse viscérale + manteau) chez les moules.

\section{Chez les moules}

La récupération des parties molles des moules se fait de façon simple à l'aide d'une pince après avoir écarté les deux valves de la coquille. Ceci laisse apparaître la masse viscérale enveloppée du manteau accolée sur l'une des valves dans le cas des moules grillées et sur les deux valves dans le cas des moules crues. A l'aide d'une lame, on coupe le muscle adducteur reliant la partie molle de la moule à la coquille. Les parties molles de chaque lot de moules sont emballées dans des sachets en plastique étiquetés et conservés dans un réfrigérateur jusqu'à analyse.

\section{Chez les oursins}

Cette opération consiste pour chaque lot d'oursins à enlever d'abord les piquants en faisant un raclage à l'aide d'une lame.

L'animal ainsi dépourvu de piquants est ensuite disposé bouche vers le haut pour une incision. Avec une paire de ciseaux, on fait une incision circulaire, horizontalement juste en bas de l'orifice buccal de façon à ne pas endommager les gonades ayant une location plus basse. Les deux parties hémisphériques sont séparées: l'une contenant la lanterne d'Aristote et l'autre partie 
renfermant les gonades, les intestins, le système nerveux, le système aquifère par exemples. Les gonades disposées en 5 masses charnues de couleur orangée sont extraites au moyen d'une pince fine et mises dans des sachets en plastiques avec des étiquettes et conservés dans le réfrigérateur jusqu'à analyse.

\section{Broyage et séchage}

Le broyage des gonades d'oursins et des masses viscérales des moules s'est fait à l'aide du broyeur électrique, Moulinex. Ainsi un mélange homogène est obtenu pour chaque espèce.

\section{Evaluation des teneurs en mercure}

Après broyage et homogénéisation, un sous-échantillon de $0,1 \mathrm{~g}$ a été pesé pour chaque échantillon pour analyse. Les concentrations de mercure (Hg) dans les sous-échantillons humides pesés ont été déterminées par un analyseur direct de mercure, le DMA-8 qui utilise le principe de la décomposition thermique, de la fusion et l'absorption atomique (Erasmus et al., 2018). Il est spécialement conçu pour déterminer le $\mathrm{Hg}$ total contenu dans les échantillons à l'état solide, liquide ou gazeux, sans traitement des échantillons. Afin d'évaluer la capacité analytique du DMA-80 utilisé, la précision et la qualité des mesures de $\mathrm{Hg}$ étaient vérifié à l'aide d'un matériau de référence certifié (CRM, AIEA-457) constitué de chair de moule avec une concentration de $0,143 \mathrm{mg} \cdot \mathrm{kg}^{-1}$ en $\mathrm{Hg}$ total, comme matériau de contrôle de la qualité (QCM) fourni par l'Agence Internationale de l'Energie Atomique (AIEA). Le taux de recouvrement était en moyenne $89 \%$ est dans l'intervalle acceptable (80\%-110\%). La limite de détection du DMA-80 est de 0,01 ng. Les analyses ont réalisées au niveau du laboratoire de chimie de l'institut de technologie alimentaire (ITA) du Sénégal.

\section{Traitement et analyse des données}

\section{Test statistiques}

Le test de Student a été effectué pour examiner la significativité de la variation temporelle des teneurs en mercure entre les produits crus et grillés pour chaque espèce, mais également entre espèce. Le logiciel statistique $\mathrm{R}$ a été utilisé pour réaliser ces tests.

\section{Evaluation des risques sanitaires}

L'évaluation des risques sanitaires liés à la consommation de ces produits a été effectuée à partir de deux indices : le quotient de risque (THQ) et la masse maximale tolérable par jour (MSC). 


\section{Quotient de risque (Target Hazard Quotient en Anglais, THQ)}

Les risques sanitaires liés à la consommation quotidienne d'aliments contaminés par des métaux traces peuvent être évalués en utilisant le quotient de risque (THQ) (Khoshnood et al, 2014 ; Diop et al., 2017 ). Cette méthode définie par USEPA (2000) est appliquée dans cette étude en vue d'apprécier les risques sanitaires liés à la consommation des moules et des oursins. Le THQ est définie par l'équation ci-contre :

$$
T H Q=\frac{E F r x \times E D t o t \times W f o o d \times C i}{R F d o x \times B w x \times A T n} \times 10^{-3}
$$

Si THQ < 1 indique un effet négligeable sur la santé humaine Si THQ> 1 indique un effet néfaste sur la santé humaine (Diop et al., 2017) EFr : Fréquence d'exposition (365jours) (Diop et al., 2017) EDtot : Espérance de vie (65 ans au Sénégal: ANSD, 2017).

Wfood : Quantité journalière consommée $(\mathrm{g})$. Elle correspond à $1000 \mathrm{~g}$ pour les moules et $200 \mathrm{~g}$ pour les oursins. Ces valeurs correspondent aux quantités maximales qu'un individu peut consommer par jour. Elles ont été définies après des enquêtes réalisées au niveau des consommateurs et vendeurs.

$\mathrm{Ci}$ : Concentration du métal $(\mathrm{mg} / \mathrm{kg})$

RFdox : Dose de référence (3x 10 $10^{-4} \mathrm{mg} / \mathrm{kg} / \mathrm{jour}$, (USEPA, 2008)

Bwx : Poids corporel moyen (60 kg) (Diop et al., 2017)

Atn : Durée d'exposition (365jours x espérances de vie)

\section{Masse journalière maximale tolérable (MSC).}

Toujours dans le but de mieux alerter les consommateurs, la masse maximale tolérable par jour pour la consommation des moules et oursins est estimée. Elle peut être déterminée à partir de la dose journalière maximale provisoire notée $\mathrm{JLi}$, de la concentration du métal (Ci) et du poids corporel $\mathrm{Bw}$ (Diop et al., 2017) selon la formule ci-dessous:

$$
M S C i=\frac{\mathrm{Ji} \times \mathrm{Bw}}{\mathrm{Ci}}
$$

$\mathrm{JLi}=$ Dose journalière maximale provisoire $(0,47 \mu \mathrm{g} / \mathrm{kg})$

$\mathrm{Bw}=$ poids corporel $(60 \mathrm{~kg})$

$\mathrm{Ci}=$ Concentration du mercure $(\mu \mathrm{g} / \mathrm{kg})$

\section{Résultats et discussion}

\section{Concentration de $\mathrm{Hg}$ chez les moules et les oursins}

Les teneurs en mercure $(\mathrm{Hg})$ obtenues sont résumées dans le tableau 1. Pour les moules, elles varient de $0,0172 \mathrm{mg} \cdot \mathrm{kg}^{-1}$ à $0,0244 \mathrm{mg} \cdot \mathrm{kg}^{-1}$ pour les produits crus contre $0,0358 \mathrm{mg} \cdot \mathrm{kg}^{-1}$ et $0,0475 \mathrm{mg} \cdot \mathrm{kg}^{-1}$ pour les produits transformés. En ce qui concerne les oursins, les teneurs en $\mathrm{Hg}$ oscillent entre $0,0153 \mathrm{mg} \cdot \mathrm{kg}^{-1}$ et $0,0195 \mathrm{mg} \cdot \mathrm{kg}^{-1}$ et entre $0,0274 \mathrm{mg} \cdot \mathrm{kg}^{-1}$ et $0,0475 \mathrm{mg} \cdot \mathrm{kg}^{-1}$ respectivement pour les produits crus et grillés. Malgré la présence de métal toxique dans ces produits, les concentrations détectées sont très en deçà de la 
norme fixée par l'agence américaine pour la protection de l'environnement (USEPA) pour les produits de la mer en 2008 qui est de $0,5 \mathrm{mg} \cdot \mathrm{kg}^{-1}$.

Tableau 1. Taux de mercure enregistrés chez les moules et les oursins en $\mathrm{mg}^{\mathrm{kg}} \mathrm{kg}^{-1}$ de poids frais.

\begin{tabular}{|l|l|l|l|l|}
\cline { 2 - 5 } \multicolumn{1}{c|}{} & Moules crues & Moule grillées & Oursins crus & Oursins grillés \\
\hline Min & 0,0172 & 0,0358 & 0,0153 & 0,0213 \\
\hline Max & 0,0244 & 0,0475 & 0,0195 & 0,0274 \\
\hline Moyenne & 0,0208 & 0,0416 & 0,0174 & 0,0243 \\
\hline Ecart type & 0,0051 & 0,0083 & 0,0030 & 0,0142 \\
\hline
\end{tabular}

La figure 3 présente les variations du taux de mercure rencontrées chez les moules et les oursins suivant les périodes d'échantillonnage.
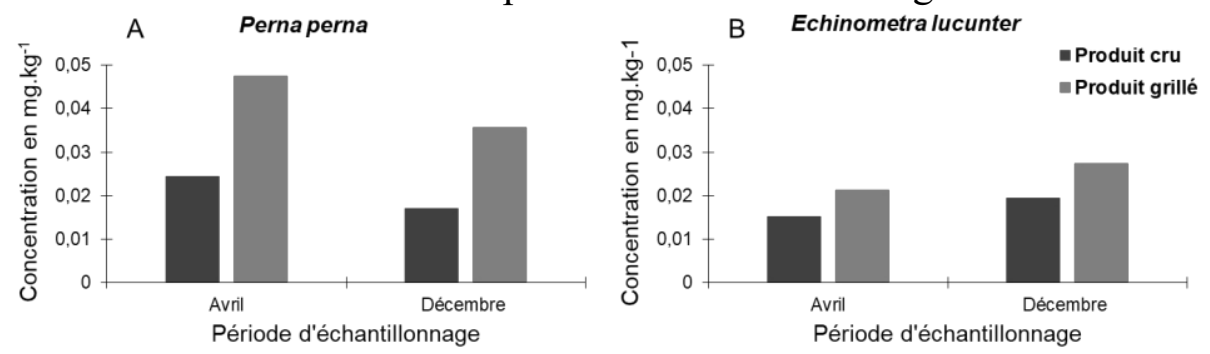

Figure 3. Variations temporelles des teneurs en mercure dans les moules (A) et dans les oursins (B).

Suivant les périodes de collecte, les teneurs en mercure les plus élevées sont observées en Avril $\left(0,0359 \pm 0,008 \mathrm{mg} \cdot \mathrm{kg}^{-1}\right)$ et les plus faibles en Décembre $\left(0,0265 \pm 0,005 \mathrm{mg} \cdot \mathrm{kg}^{-1}\right)$ chez les moules, tandis que chez les oursins les teneurs les plus importantes sont enregistrées en Décembre avec $0,0234 \pm 0,004 \mathrm{mg} \cdot \mathrm{kg}^{-1}$ contre 0,0183 $\pm 0,002 \mathrm{mg} \cdot \mathrm{kg}^{-1}$ en Avril. Ces résultats peuvent être expliqués par l'influence de la reproduction sur la physiologie de ces espèces. En effet, la reproduction a lieu pour les moules et oursins respectivement en Avril et en Décembre (Gayré, 1978 ; Lewis \& Storey, 1984). Durant ces périodes, les individus adultes se trouvent à leur optimum de développement sexuel (His \& Cantin, 1992). Ce qui se traduit par une augmentation de l'apport alimentaire qui constitue leur principale voie de contamination (Augier et al., 1997). Cependant, tant pour les moules que pour les oursins, le test de Student réalisé signale que ces différences de concentrations observées ne sont pas statistiquement significatives $(p>0,05)$. Concernant la nature des produits, il apparait que aussi bien chez les moules que chez les oursins, les teneurs en mercure $(\mathrm{Hg})$ sont nettement plus élevées dans les produits grillés quelle que soit la période d'échantillonnage. En moyenne quelle que soit l'espèce les produits grillés contiennent deux fois plus de mercure que les produits crus. Ces écarts de concentrations entre les 
produits crus et grillés observés dans cette étude sont aussi observés par d'autres auteurs. Ouédraogo \& Amyot (2011) ont montré que la cuisson et la friture ont un impact sur le niveau de contamination du mercure chez le thon. Selon Do et al. (2015) les différences de concentration de mercure dans les produits crus et grillés, peuvent être expliquées par deux phénomènes. Une perte d'eau lors de la grillade qui provoque une diminution de l'humidité ; cela peut conduire d'une part à l'augmentation des protéines amenant ainsi le mercure à se concentrer plus fortement dans les tissus (Morgan et al, 1997) et d'autre part à la formation de complexes impliquant des formes de mercure et des groupes sulfhydriles présents dans les tissus.

\section{Evaluation des risques sanitaires}

L'évaluation des risques de santé liés à la consommation des moules et des oursins est effectuée grâce à deux indices (THQ et MSC).

\section{Quotient de risque (THQ)}

Dans le tableau 2 figurent les valeurs de THQ obtenues chez les moules et les oursins.

Tableau 2. Valeurs de THQ obtenues pour un adulte de $60 \mathrm{~kg}$.

\begin{tabular}{|l|l|l|l|}
\hline Produits & Types & Moyennes des Taux de Hg en mg.kg & THQ \\
\hline \multirow{3}{*}{ Perna perna } & Crues & 0,0208 & 0,421 \\
\cline { 2 - 4 } & Grillées & 0,0416 & 0,844 \\
\hline \multirow{2}{*}{$\begin{array}{l}\text { Echinometra } \\
\text { lucunter }\end{array}$} & Crus & 0,0174 & 0,070 \\
\cline { 2 - 4 } & Grillés & 0,0243 & 0,098 \\
\hline
\end{tabular}

La totalité des valeurs de (THQ) calculées sont inférieures à 1 (valeur référence). Cela implique que les risques sanitaires liés à la consommation des moules et oursins sont négligeables pour un individu adulte. Le THQ est plus élevé au niveau des produits grillés quelle que soit l'espèce. Il est plus important chez les moules. Chez ces dernières, le THQ varie entre 0,421 (moules crues) et 0,844 (moules grillées), alors qu'il oscille entre 0,070 et 0,098 respectivement pour les oursins crus et grillés. Cependant même si les valeurs de THQ obtenues sont en dessous de 1, il est important de souligner que les moules grillées présentent un THQ $(0,8445)$ très proche de cette valeur de référence ce qui constitue une menace pour la santé des consommateurs de moules grillées. Selon l'OMS, une exposition chronique au mercure peut causer des troubles neurologiques et une altération du fonctionnement des reins (WHO, 2003). 


\section{Masse maximale tolérable (MCS)}

Les masses journalières maximales tolérables liées à la consommation de ces produits sont résumées dans le tableau 3.

Tableau 3. Evaluation de la masse maximale tolérable (MSC) par semaine des produits contaminés pour un adulte de $60 \mathrm{~kg}$.

\begin{tabular}{|l|l|l|l|}
\hline Produits & Types & Moyennes des Taux de Hg en mg.kg-1 & MSC $\left(\mathrm{kg} . \mathrm{j}^{-1}\right)$ \\
\hline \multirow{2}{*}{ Perna perna } & Crues & 0,0208 & 1,355 \\
\cline { 2 - 4 } & Grillées & 0,0416 & 0,677 \\
\hline \multirow{2}{*}{$\begin{array}{l}\text { Echinometra } \\
\text { lucunter }\end{array}$} & Crus & 0,0174 & 1,620 \\
\cline { 2 - 4 } & Grillés & 0,0243 & 1,160 \\
\hline
\end{tabular}

Ces valeurs oscillent entre $0,677 \mathrm{~kg} \cdot \mathrm{j}^{-1}$ (moules grillées) et $1,355 \mathrm{~kg} \cdot \mathrm{j}^{-}$ ${ }^{1}$ (moules crues). Concernant les oursins le MSC est au environ de 1 (1,160 $\mathrm{kg} . \mathrm{j}^{-1}$ pour les oursins grillés et $1,620 \mathrm{~kg} . \mathrm{j}^{-1}$ pour les oursins crus). Les valeurs plus faibles de MSC chez les produits grillés sont dues fortes concentrations de mercure rencontrées dans ces produits. Autrement dit, plus la concentration de mercure est élevée, plus le MSC est faible. Les valeurs obtenues dans cette étude sont 3 à 4 fois plus faibles que celles trouvées par Diop et al., (2017). Ceci est dû au fait que dans cette étude, les concentrations en mercure rencontrées dans les produits sont plus élevées que celles de Diop et al., (2017).

\section{Conclusion}

La présente étude a porté sur la détermination des concentrations de mercure dans les parties comestibles des moules et oursins commercialisés au niveau de Soumbédioune et les risques sanitaires liés à leur consommation. Elle révèle la présence de ce métal toxique dans ces produits fortement consommés par les populations, en particuliers les jeunes qui se baignent sur la plage de Soumbédioune. Les teneurs en mercure sont plus importantes chez les moules. L'étude des variations temporelles de teneurs en mercure indique qu'elles sont plus élevées chez les moules en avril, alors que chez les oursins elles sont maximales en décembre. En outre, suivant les formes (crues ou grillées), les produits grillés renferment plus de mercure. Toutefois, ces teneurs en mercure enregistrées dans ces produits sont largement inférieures à la norme fixée par USEPA en 2008. L'évaluation des risques sanitaires à travers le quotient de risque (THQ) et le MSC montre que globalement la consommation de ces produits ne présente aucune menace pour le moment la santé publique. Cependant, Il convient de consommer les moules et les oursins avec beaucoup de modération car une exposition chronique au mercure peut avoir des conséquences dramatiques sur la santé. 
En raison des risques écotoxicologiques et sanitaires liés à la pollution métallique et à la consommation des moules et des oursins au Sénégal, cette présente étude mérite d'être approfondie et élargie aux autres métaux traces non essentiels aux processus biologiques comme le plomb, le cadmium et l'arsenic.

\section{References :}

1. ANSD (2019). Rapport de la Situation Economique et Sociale du Sénégal en 2016.

2. Augier, H., Desmerger, R., Egèa, M., lmbert, E., Park, WK., Ramonda, G., Santimone, M. (1997). Étude à l'aide de bio-indicateurs (oursins et moules) de la pollution des ports de plaisance par les métaux traces sur le littoral provençal. Mar. Life, Vol. 7 (1-2): 67-81.

3. Bell, L., DiGangi, J., Weinberg, J. (2014). Manuel d'introduction pour l'ONG à la pollution par le mercure et la convention de Minamata sur le mercure, $249 \mathrm{p}$.

4. Bélanger, D. (2009). Utilisation de la faune macrobenthique comme bioindicateur de la qualité de l'environnement marin côtier. Mémoire de master 2, Ecologie internationale. Sherbrooke, Québec : Université de Sherbrooke, $74 \mathrm{p}$.

5. CCLME (2014). Programme d'action mondiale pour la protection du milieu marin contre la pollution due aux activités terrestres, le cas du Sénégal, 51p.

6. Diop, M., Howsam, M., Diop, C., Goossens, JF., Diouf A., Amara, R. (2016). Assessment of trace element contamination and bioaccumulation in algae (Ulva lactuca), mussels (Perna perna), shrimp (Penaeus kerathurus), and fish (Mugil cephalus, Saratherondon melanotheron) along the Senegalese coast. Marine Pollution Bulletin, n'106: 339-343.

7. Diop, M., Net, S., Howsman, M., Lencel, P., Watier, D., Grard, T., Duflos, G., Amara, R. (2017). Concentration and potential human health risks for regulated metals $(\mathrm{Cd}, \mathrm{Pb}, \mathrm{Hg})$ and organic polluants (PAHs, PcBs) in fish and sea food from the Senegalese coast. International Journal of Environnement, Vol. 11, $\mathrm{n}^{\circ} 3$ :11-27.

8. Diouf, SM. (2017). Etude de la contamination de l'aire marine protégée de Joal-Fadiouth par le mercure. Mémoire de master II, Institut universitaire de pêche et d'aquaculture. Dakar: Université Cheikh Anta Diop, 34p.

9. Do, F., Costa, N., Korn, MDG. (2015). Résultats préliminaires des concentrations de mercure dans les fruits de mer crus et cuits et leur impact sur la santé publique. Food Chemistry, Vol.81, nº ${ }^{\circ}$ 192- 837. 
10. Erasmus, VN., Hamutenya, S., Iitembu, JA. (2018). Mercury concentrations in muscles and liver tissues of Cape monkfish (Lophius vomerinus) from the Northern Benguela, Namibia. Marine Pollution Bulletin 135: 1101-1106

11. Gayré, P. (1978). Etude de la moule Perna et des possibilités de mytiliculture en république populaire du Congo. Série océanogr., vol.16. $\mathrm{n}^{\circ} 1$ : 9-17.

12. His, E., Cantin., C. (1992). Biologie et physiologie des coquillages. Publication IFREMER, 118p.

13. Lewis, JB., Storey, GS. (1984). Differences in morphology and life history traits of the echinoid Echinometra lucunter from different habitats. Marine Ecology-Progress Series, Vol.15: 207-211.

14. Marusczak, N. (2010). Etude du transfert du mercure et du méthylmercure dans les écosystèmes lacustres alpins. Thèse de doctorat, Sciences de la Terre et de l'Univers et de l'Environnement. Université de Grenoble, 207p.

15. Metongo,BS.(1991).Concentrations en metaux toxiqueschez Crassostrea gasar (huitre de mangrove) en zone urbaine lagunaire d'abidjan (Côte d'Ivoire). Journal.Océano.Limnol.AbidjanVol.1:3345.

16. Ouédraogo $\mathrm{O} \&$ Amyot $\mathrm{M}$. Effects of various cooking methods and food components on bioaccessibility of mercury from fish. Environnmental rechearch, 2011, Vol.111, nº : 10-1016.

17. Scheuhammer, AM., Basu, N., Evers, DC., Heinz, GH., Sandheinrich, MB., and Bank, MS. (2012). Ecotoxicology of mercury in fish and wildlife dans : Bank, MS. (Ed) Mercury in the Environment - Pattern and Process, University of California Press, Berkeley and Los Angeles, California, USA, pp 223-238. 